\title{
Adaptive Virtual MIMO Single Cluster Optimization in a Small Cell
}

\author{
Triantafyllos Kanakis, Michael Opoku Agyeman, Anastasios Bakaoukas \\ Computing Department, Faculty of Arts, Science \& Technology, University of Northampton, UK \\ Email: \{triantafyllos.kanakis, michael.opokuagyeman, anastasios.bakaoukas\}@ northampton.ac.uk
}

\begin{abstract}
Adaptive Virtual MIMO optimized in a single cluster of small cells is shown in this paper to achieve near Shannon channel capacity when operating with partial or no Channel State Information. Although, access links have enormously increased in the recent years, the operational system complexity remains linear regardless of the number of access nodes in the system proposed.

Adaptive Virtual MIMO optimized in a single cluster performs a theoretical information spectral efficiency, almost equal to that of the upper bounds of a typical mesh network, up to 43 bits/s/Hz at a SNR of $30 \mathrm{~dB}$ while the BER performance remains impressively low hitting the $10^{-6}$ at an SNR of about 13 dB when the theoretical upper bound of an ideal small cell mesh network achieves the $10^{-6}$ at a SNR of $12.5 \mathrm{~dB}$. In addition, in a sub-optimum channel condition, the channel capacity and BER performance of the proposed solution is shown to drastically delay saturation even for the very high SNR.
\end{abstract}

Index Terms-Adaptive Multiuser Detection, MIMO, Small Cell, Single Cluster, Inter-cell Interference, Partial Channel State Information.

\section{INTRODUCTION}

Wireless and mobile communication networks have been massively transformed in the past decade. The constantly increasing number of data hungry devices have forced cellular network providers to often deliver data traffic in excess of $95 \%$ of the time. At the same time M2M and IoT have significantly changed the number of devices in a network while the access radio communication macro-cells are expected to be proved short in providing for the demand in due time. The industry and research community now recognise that channel capacity gains can only be achieved by spectrum reuse. Based on this principle Small Cells have been introduced increasing the dimensionality of the cellular networks providing short range connectivity for a large number of devices. In addition, with the growing demand for large data, new ways to increase bandwidth efficiency are necessary.

Multiple antenna systems benefit from spatial diversity resulting in spectral efficiency increase [1][2][3][4]. Spacing between the antennas within a single communication node is normally expected larger than half-wavelength [5], however due to the small physical dimensions of the access devices this is not always possible and when it is, the number of antennas on a single communication node will be small. This limitation is solved by the use of virtual antenna arrays as shown in [6]. Finally, centralized multiuser detection schemes as proposed in [7], are satisfactorily capable to keep intra-cell interferers under precise power control but the inter-cell interference is fully out of their control.

From this point on the term cooperative wireless communication system will refer to a fully interference controllable cooperative system where all communication terminals have full knowledge of:

- The channel statistics,

- The exact number of users $K$

while no unknown interfering nodes $K_{i}$ will transmit in the area $\left(K_{i}=0\right)$. This is an ideal channel condition which is without a doubt very unlikely to happen in actual deployments and is used in this paper for comparison purposes only. Therefore, the term sub-cooperative wireless communication system will refer to an interference non-controllable cooperative system where the communication terminals have only limited or often no knowledge of:

- the statistics of the channel

- the exact number of users $K$

- the number of interfering nodes $K_{i}$, where $\left(K_{i} \neq 0\right)$

A sub-optimum yet more realistic system as described above will be considered throughout this paper.

Adaptive Virtual MIMO (AV-MIMO) in a Single Cluster Optimization (SCO) is shown in this paper to be used with small cells to increase spectral efficiency in the local cluster without the need of multiple antennas on each individual access device. Instead AV-MIMO-SCO makes use of the Adaptive Multiuser Detection (AMUD) as shown in [8][9] to utilize multiple communication nodes equipped with a singleantenna into a multi-antenna node equivalent to this of a Virtual Antenna Array (VAA) as shown in [10][11]. An AVMIMO system is inherently (at the physical layer) responding to a time variable nature of the VAA [10] environment. Apart from backhauling, the small cell acts as a simple access device and is not always in the formation of the Adaptive Virtual Antenna Array (AVAA).

An AV-MIMO-SCO sub-cooperative system achieves a normalized information spectral efficiency of $28.75 \mathrm{bits} / \mathrm{s} / \mathrm{Hz}$ at a SNR of $30 \mathrm{~dB}$ and a BER of $10^{-6}$ at a SNR of about $25.75 \mathrm{~dB}$ in a cooperative environment. In a sub-cooperative environment (with 4 unknown interferers), the sub-cooperative AV-MIMO-SCO system putting through a system channel capacity of $25 \mathrm{bits} / \mathrm{s} / \mathrm{Hz}$ at a SNR of $30 \mathrm{~dB}$ where the cooperative system does not exceed the $15.5 \mathrm{bits} / \mathrm{s} / \mathrm{Hz}$. In the sub-cooperative environment, the AV-MIMO-SCO achieves a BER of $10^{-6}$ at a SNR of about $28.5 \mathrm{~dB}$ while the cooperative 
system saturates at a BER of $10^{-3}$ at a SNR of $30 \mathrm{~dB}$.

\section{Communication Protocol}

For simplicity, the coverage of a cluster should be considered the transmission coverage of the small cell while $S_{k}$ number of users operate within the cluster.

1. Phase I: Constant Operation All users broadcast a training sequence message with length of $\mathrm{M}$ data symbols. Upon reception of the neighbors' training sequences and by means of Minimum Mean Square Error (MMSE) estimation [12] [9], nodes get to increase statistical knowledge of the channel with each neighbor node in the cluster.

2. Phase II: AVAA formation By means of a very small data packet sent with the training sequence, the source node informs the destination node of its intention to send data packets. From the constant operation shown in PhaseI source and destination nodes activate the neighbor nodes that provided them with the best MMSE during the latest training sessions and they form the local AVAAs as shown in Fig.1 (source and destination communication nodes in black).

3. Phase II: AV-MIMO operation The source node forms a short range (low power) broadcast channel and distributes information packets to the nodes contributing to the transmit AVAA. The $M$ transmit AVAA nodes form a $N \times M$ dimensional MIMO channel with the $N$ receive AVAA nodes. The information packets are spread and transferred to the receiver AVAA through the MIMO channel.

4. Phase IV: Information recovery The receiver AVAA forwards the received information packets to the destination node in a single-cast low power operation. At the destination node the channels are de-correlated using AMUD [12] [9] forming an unbiased estimator for the information packets.

\section{System Model}

The system is similar to this in [6] with a total of $K$ operational nodes. The nodes "recruited" by the source and destination are denoted in gray in Fig.1. This forms a $M \times N$ AV-MIMO system where

$$
\sum_{k=2}^{K}(M, N) \leq K
$$

where $M$ is the dimension of the transmitter AVAA, $N$ is the dimension of the receiver AVAA and $K$ is the total number of nodes within the system.

The signal at every output of the communications channel of the system follows the standard linear type

$$
\mathbf{y}=\mathbf{H x}+\mathbf{n}
$$

where $\mathbf{x}$ is the information signal, $\mathbf{H}$ is a composite channel, $\mathbf{n}$ is the noise vector and $\mathbf{y}$ is the signal at the destination node.

The recruited nodes on each side along with the source and destination nodes respectively, team up to form an $M \times N$ AVAA system.

The linear system model with respect to Fig. 1 and by assuming negligible noise within the local cluster:

$$
\mathbf{y}_{1}=\mathbf{A}_{0} \mathbf{x}+\mathbf{n}_{0}
$$

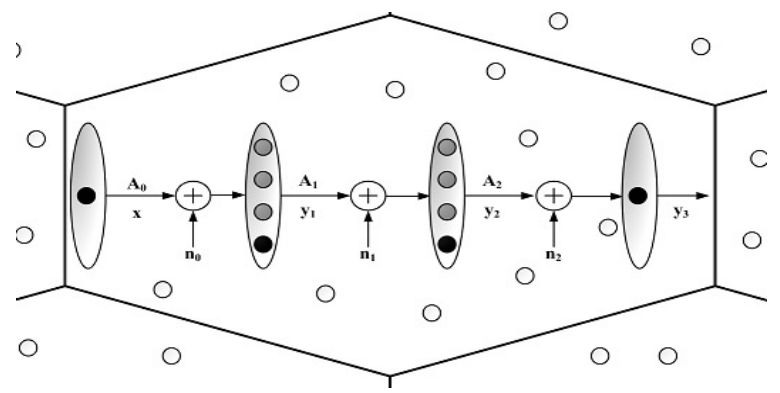

Fig. 1. General system model of AV-MIMO

where $\mathbf{x}$ is the original information vector, $\mathbf{A}_{0}$ is the broadcast channel of the transmit AVAA, $\mathbf{n}_{0}$ is the noise vector and $\mathbf{y}_{1}$ is the signal vector at the receiver AVAA.

The broadcast channel $\mathbf{A}_{0}$ is represented by a diagonal matrix of Zero Mean Circular Symmetric Complex Guassian (ZMCSCG) Independent and Identically Distributed (i.i.d.) random variable coefficients with variance of 1 [1]:

$$
\mathbf{A}_{0}=\operatorname{diag}\left[\begin{array}{llll}
\alpha_{1} & \alpha_{2} & \cdots & \alpha_{M}
\end{array}\right]
$$

where all sub-channels of $\mathbf{A}_{0}$ are orthogonal to each other. The source and destination nodes are always participating to the formed AVAA.

The linear system model with respect to Fig. 1, by assuming that noise influences the transmission and by equation 2 :

$$
\begin{gathered}
\mathbf{y}_{2}=\mathbf{S A}_{1} \mathbf{y}_{1}+\mathbf{n}_{1} \\
\mathbf{y}_{2}=\mathbf{S A}_{1}\left(\mathbf{A}_{0} \mathbf{x}+\mathbf{n}_{0}\right)+\mathbf{n}_{1}
\end{gathered}
$$

where $\mathbf{A}_{1}$ is the MIMO channel, $\mathbf{S}$ is the CDMA spreading codes matrix, $\mathbf{n}_{1}$ the noise vector and $\mathbf{y}_{2}$ is the signal at the receiver AVAA.

For the MIMO channel shown in Fig. 1, $M$ active transmitters and $N$ active receivers are assumed where $M \leq n_{t}$ and $N \leq n_{r}$. The MIMO channel $\mathbf{A}_{1}$ is a $(N \times M)$ matrix with ZMCSCG i.i.d. random variables where coefficients have a variance of 1 as follows:

$$
\mathbf{A}_{1}=\left[\begin{array}{cccc}
\beta_{1,1} & \beta_{1,2} & \ldots & \beta_{1, M} \\
\beta_{2,1} & \beta_{2,2} & \ldots & \beta_{2, M} \\
\vdots & \vdots & \ddots & \vdots \\
\beta_{N, 1} & \beta_{N, 2} & \cdots & \beta_{N, M}
\end{array}\right]
$$

Information packets are forwarded to the destination node through $\mathbf{A}_{2}$ MISO channel.

The linear model with respect to Fig. 1 will be

$$
\begin{gathered}
\mathbf{y}_{3}=\mathbf{A}_{2} \mathbf{y}_{2}+\mathbf{n}_{2} \\
\mathbf{y}_{2}=\mathbf{A}_{2} \mathbf{S} \mathbf{A}_{1} \mathbf{A}_{0} \mathbf{x}+\mathbf{A}_{2} \mathbf{n}_{1}
\end{gathered}
$$

where $\mathbf{A}_{2}$ is the MISO channel and $\mathbf{y}_{3}$ is the signal at the destination node. $\mathbf{A}_{2}$ is a MISO diagonal matrix with ZMCSCG i.i.d. random variables with unit variance coefficients [1] as follows:

$$
\mathbf{A}_{2}=\operatorname{diag}\left[\begin{array}{llll}
\gamma_{1} & \gamma_{2} & \cdots & \gamma_{M}
\end{array}\right]
$$


For the purpose of analysis the following parameters are set:

$$
\mathbf{y}=\mathbf{H x}+\mathbf{N}
$$

where,

$$
\begin{gathered}
\mathbf{H}=\mathbf{A}_{2} \mathbf{S} \mathbf{A}_{1} \mathbf{A}_{0} \\
\mathbf{N}=\mathbf{A}_{2} \mathbf{n}_{1}
\end{gathered}
$$

\section{Mean Square Error Analysis}

In order to simplify calculations in the estimation of the overall system channel capacity a set of definitions have been introduced in [6].

Definitions :

1) Autocovariance matrix $\mathbf{R}=\mathcal{E}\left\{\mathbf{y} \mathbf{y}^{H}\right\}$

2) Crosscorrelation vector $\mathbf{p}=\mathcal{E}\left\{x_{k}^{*} \mathbf{y}\right\}$

3) Filter output statistic $\hat{x}_{k}(\mathbf{w})=\mathbf{w}^{H} \mathbf{y}$

where $\mathcal{E}(\bullet)$ typify the expectation function, $(\bullet)^{H}$ represents the Hermitian matrix (complex conjugate and transpose) and $x_{k}$ is the training sequence of the $k^{t h}$ user where $k \leq K$. Finally, $\mathbf{y}$ is the signal received at the destination node and $\mathbf{w}$ introduce the matched filter coefficients. by

As shown in [6], at the destination node, the error is given

$$
J(\mathbf{w})=\mathcal{E}\left\{\left|x_{k}-\hat{x}_{k}(\mathbf{w})\right|^{2}\right\}
$$

where, expanding equation (11) yields

$$
J(\mathbf{w})=\mathcal{E}\left\{x_{k}^{*}\left(x_{k}-\hat{x}_{k}(\mathbf{w})\right)\right\}-\mathcal{E}\left\{\hat{x}_{k}^{*}(\mathbf{w})\left(x_{k}-\hat{x}_{k}(\mathbf{w})\right)\right\}
$$

Lemma 1 : For MMSE $\mathcal{E}\left\{\hat{x}_{k}^{*}(\mathbf{w})\left(x_{k}-\hat{x}_{k}(\mathbf{w})\right)\right\}=0$ where the expectation of $\hat{x}_{k}(\mathbf{w})$ is orthogonal to the error.

Proof :

$\mathcal{E}\left\{\left(\mathbf{w}^{H} \mathbf{y}\right)^{*}\left(x_{k}-\mathbf{y}^{H} \mathbf{w}\right)\right\}=\mathcal{E}\left\{\mathbf{w}^{H} \mathbf{y} x_{k}^{*}\right\}-\mathcal{E}\left\{\mathbf{w}^{H} \mathbf{y} \mathbf{y}^{H} \mathbf{w}\right\}$

Since $\mathbf{w}$ is constant, then

$$
\begin{gathered}
\mathcal{E}\left\{\left(\mathbf{w}^{H} \mathbf{y}\right)^{*}\left(x_{k}-\mathbf{y}^{H} \mathbf{w}\right)\right\}=\mathbf{w}^{H} \mathcal{E}\left\{\mathbf{y} x_{k}^{*}\right\}-\mathbf{w}^{H} \mathcal{E}\left\{\mathbf{y} \mathbf{y}^{H}\right\} \mathbf{w} \\
\mathcal{E}\left\{\left(\mathbf{w}^{H} \mathbf{y}\right)^{*}\left(x_{k}-\mathbf{y}^{H} \mathbf{w}\right)\right\}=\mathbf{w}^{H} \mathbf{p}-\mathbf{w}^{H} \mathbf{R} \mathbf{w}
\end{gathered}
$$

Corollary 1 : For the MMSE $\mathbf{w}$ is given by $\mathbf{w}=\mathbf{R}^{-1} \mathbf{p}$. By substitution

$$
\mathbf{w}^{H} \mathbf{p}-\mathbf{w}^{H} \mathbf{R} \mathbf{w}=0
$$

So, for MMSE $\mathcal{E}\left\{\hat{x}_{k}^{*}(\mathbf{w})\left(x_{k}-\hat{x}_{k}(\mathbf{w})\right)\right\}=0$. When $\mathbf{w}=\mathbf{R}^{-1} \mathbf{p}$, it is clear that

$$
J(\mathbf{w})=\mathcal{E}\left\{x_{k}^{*}\left(x_{k}-\hat{x}_{k}(\mathbf{w})\right)\right\}
$$

Given Lemma $1 J(\mathbf{w})=\sigma_{x}^{2}-\mathbf{w}^{H} \mathbf{p}$, therefore,

$$
J(\mathbf{w})=\sigma_{e}^{2}=\sigma_{x}^{2}-\mathbf{p}^{H} \mathbf{R}^{-1} \mathbf{p}
$$

where $\sigma_{e}^{2}$ is the error variance. $J(\mathbf{w})$ is the error function, where the Minimum Mean Square Error is for $\mathbf{w}=\mathbf{R}^{-1} \mathbf{p}$.

\section{AMUD with Least Mean SQUares}

The Least Mean Square (LMS) algorithm as well known to converge to the MMSE within a small arbitrary constant [9]. The LMS is given as:

$$
\mathbf{w}_{k+1}=\mathbf{w}_{k}+\alpha e_{k}^{*} \mathbf{y}
$$

where $e_{k}^{*}$ is the complex conjugate of the instantaneous error while $\alpha$ is the step size shown in [9] [6] to be bounded by:

$$
\begin{gathered}
0<\alpha<\frac{2}{\lambda_{\max }} \\
\sum_{i=1}^{2 G+1} \frac{\alpha \lambda_{i}}{2-\alpha \lambda_{i}}<1
\end{gathered}
$$

where $\lambda_{i}$ is the $i^{\text {th }}$ eigenvalue of the autocorrelation matrix $\mathbf{R}, \lambda_{\max }$ is the maximum eigenvalue, $2 G+1$ is the number of filter coefficients and $G$ is the length of the spreading sequence.

As shown in [9] a steady state MSE for the LMS algorithm can be given by:

$$
e(\infty)=\frac{J(\mathbf{w})}{1-\sum_{i=1}^{2 M+1} \frac{\alpha \lambda_{i}}{2-\alpha \lambda_{i}}}
$$

Given that $\alpha$ is relatively small, the steady state error is almost equivalent to $J(\mathbf{w})$.

\section{Channel Capacity}

\section{A. MIMO Capacity Upper Bound}

Channel capacity is defined in [13] as the maximum data rate assuming Gaussian input signalling with vanishingly small error probability. The factor $\frac{1}{2}$ is removed from the capacity formula and this notation is kept throughout the paper whereas all logarithms are base 2 , i.e. $\log _{2}(\bullet)$, unless differently stated.

The MIMO channel capacity formula is found in [1][14]:

$$
C=\log \left|\mathbf{I}_{r}+\hat{\mathbf{H}} \mathbf{Q} \hat{\mathbf{H}}^{H}\right|
$$

where $\mathbf{Q}=\mathcal{E}\left|\mathbf{x x}^{\mathcal{H}}\right|$ and $\operatorname{tr}(\mathbf{Q}) \leq P$. For simplicity it is assumed that $\mathbf{H}$ will be the system's composite channel.

\section{B. MMSE Capacity Analysis}

The AV-MIMO-SCO system capacity is calculated according to equation (18).

Lemma 2 : The capacity of the AV-MIMO-SCO system with a bank of MMSE filters is shown in [8] to be given by

$$
C=I_{\text {max }}\left(x_{k} ; \hat{x}_{k}(\mathbf{w})\right)=\log \left(\frac{\mathcal{E}\left\{x_{k}^{2}\right\}}{\mathcal{E}\left\{e^{2}\right\}}\right)=\log \left(\frac{1}{\sigma_{e}^{2}}\right)
$$

where $x_{k}$ is $N\left(0, \sigma^{2}\right)$.

Proof : As per Lemma 1, when a signal vector with $\mathbf{w}_{\text {opt }}$ coefficients is chosen to minimize mean error, the error signal is orthogonal to the symbol estimate $\left(\mathcal{E}\left\{\hat{x}_{k}^{*}(\mathbf{w})\left(x_{k}-\hat{x}_{k}(\mathbf{w})\right)\right\}=0\right)$. Now if $x_{1}, x_{2}, \ldots, x_{k}$ are ZMCSCG i.i.d. random variable coefficients, then:

1) $\hat{x}_{k}(\mathbf{w})$ is also Gaussian 
2) $\left(\hat{x}_{k}(\mathbf{w})-x_{k}\right)$ is Gaussian with zero mean

Lemma 3 : [13] Given that $p(x)$ follows a Gaussian distribution, then the maximum entropy $H(x)=\sigma_{x} \log (\sqrt{2 \pi e})$.

Lemma 4 : [15] The entropy of two independent random variables $\alpha$ and $\beta$ :

$$
H(\alpha \mid \beta)=H(\alpha)
$$

Lemma $5:$ : 15] For any two independent random variables $\alpha$ and $\beta$

$$
H(\alpha, \beta \mid \beta)=H(\alpha \mid \beta)
$$

Considering Lemmas 1-5, the mutual information is given by:

$$
I\left(x_{k} ; \hat{x}_{k}(\mathbf{w})\right)=H\left(x_{k}\right)-H\left(x_{k} \mid \hat{x}_{k}(\mathbf{w})\right)
$$

From Lemma 5

$$
\begin{gathered}
I\left(x_{k} ; \hat{x}_{k}(\mathbf{w})\right)=H\left(x_{k}\right)-H\left(x_{k}-\hat{x}_{k}(\mathbf{w}) \mid \hat{x}_{k}(\mathbf{w})\right) \\
I_{\max }\left(x_{k} ; \hat{x}_{k}(\mathbf{w})\right)=\log \left(\sigma_{x}^{2}\right)-\log \left(\mathcal{E}\left(n_{e}^{2}\right)\right)
\end{gathered}
$$

From Lemma 2 and considering a signal power normalized to unit energy, $\sigma_{x}^{2}=1$

$$
I_{\max }\left(x_{k} ; \hat{x}_{k}(\mathbf{w})\right)=C=\log \left(\frac{1}{\sigma_{e}^{2}}\right)
$$

By substitution of 14 to 21

$$
\begin{gathered}
C=\log \left(\frac{1}{1-\mathbf{p}^{H} \mathbf{R}^{-1} \mathbf{p}}\right) \\
0 \leq \mathbf{p}^{H} \mathbf{R}^{-1} \mathbf{p}<1
\end{gathered}
$$

for standard MMSE approach [7] where autocovariance matrix $\mathbf{R}$ and crosscorrelation vector $\mathbf{p}$ are defined in Section IV.

\section{Simulation System Model}

SNR term, as used throughout this paper, denotes the signal to thermal noise power ratio. For the Bit Error Probability results, normalized (unit energy) BPSK modulated signal is used. The CDMA spreading sequences are random Bernoulli (antipodal) sequences with a spreading factor of 32. The propagation / fading channels are modeled as normalized (unit energy) ZMCSCG i.i.d. random variables.

The total number of access nodes within the small cell cluster is arbitrarily set to 30 (i.e. $K=30$ ). The number of access nodes at the transmit and receive AVAAs is set to 4 (i.e. $M=N=4)$. The source and destination nodes are by default (as shown in the methodology) one of the four access nodes in each AVAA. Therefore, by means of MMSE estimation, the source and destination nodes activate 3 access nodes each to form their AVAAs. Hence, we end up with a virtual $4 \times 4$ virtual MIMO communication system.

When the environment is sub-cooperative, i.e. an uncontrolled form of interference present in the system, each interferer is modeled as Gaussian random variable with variance set to 0.1 (assuming that the system bandwidth is ubiquitous). This means that each interferer has precisely one tenth of the

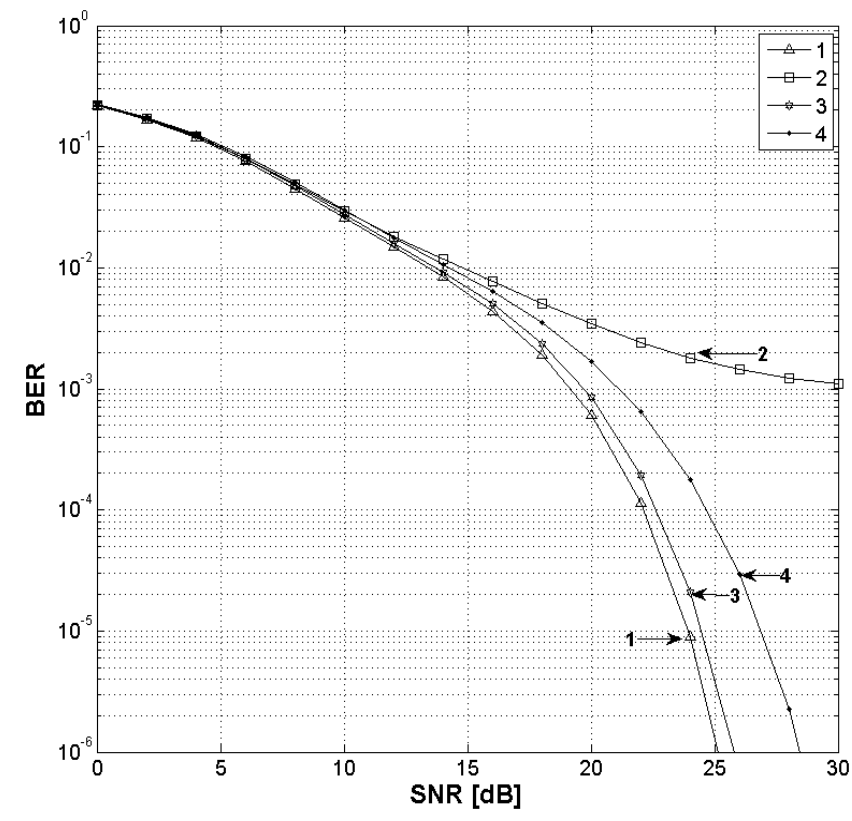

Fig. 2. BER Comparison - MSE user selection.

1) Cooperative Upper Bound - No interference.

2) Sub-Cooperative Upper Bound - Four interferers at $10 \%$ intercell power.

3) Cooperative AV-MIMO-SCO - No interferer.

4) Sub-Cooperative AV-MIMO-SCO - Four interferers at $10 \%$ intercell power.

transmit power of the AVAA. The results assume 4 independent interferers. It is well known that the AMUD techniques are able to mitigate Multiple Access Interference and thus AV-MIMO remains tolerant to many concurrent transmissions [9][16][17][18].

\section{NUMERICAL RESULTS}

As shown in Fig. 2 a BER of $10^{-6}$ is achieved for the cooperative theoretical upper bound system at a SNR of almost $25 \mathrm{~dB}$ where under the same conditions the AV-MIMO-SCO system achieves nearly the same performance with a BER of $10^{-6}$ at a SNR of $25.75 \mathrm{~dB}$. There is a small outage $(3 \%)$ due to the time delay spent to distribute data packets to all transmitting communication nodes, at the first burst. In the sub-optimum (sub-cooperative) environment a BER of $10^{-6}$ is obtained at a SNR of $28.5 \mathrm{~dB}$ by the the AV-MIMO-SCO system, which is a significant improvement over the theoretical upper bound system operating under the conditions of a subcooperative environment where the system saturates at a high BER. It is clearly shown that the AV-MIMO-SCO loses almost $15 \%$ of its energy but it is still proven more tolerant to intercell interference while the theoretical upper bound system cannot achieve a BER better than $10^{-3}$ at a SNR of $30 \mathrm{~dB}$.

At the same time, as shown in Fig. 3 in a cooperative environment, a channel capacity of $29.3 \mathrm{bits} / \mathrm{s} / \mathrm{Hz}$ is achieved at a SNR of $30 \mathrm{~dB}$ while under the same environment assumption, the AV-MIMO-SCO system achieves a spectral efficiency of $28.75 \mathrm{bits} / \mathrm{s} / \mathrm{Hz}$ at the same SNR experiencing 


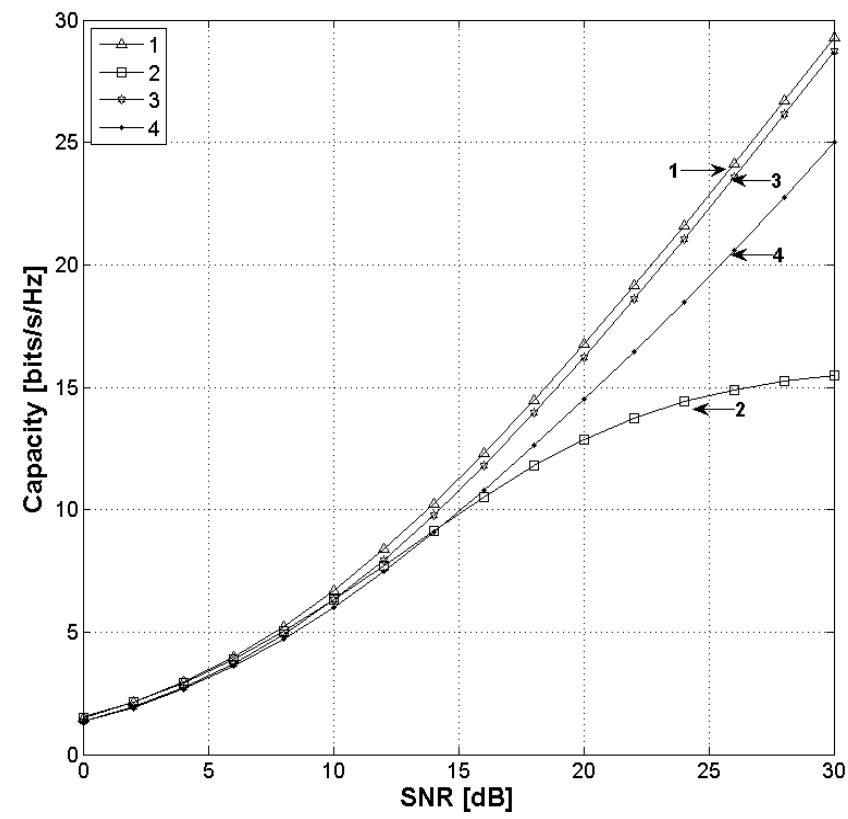

Fig. 3. Capacity Comparison - MSE user selection.

1) Cooperative Upper Bound (eq. 17) - No interference.

2) Sub-Cooperative Upper Bound (eq. 17) - Four interferers at $10 \%$ intercell power.

3) Cooperative AV-MIMO-SCO (eq. 22) - No interferer.

4) Sub-Cooperative AV-MIMO-SCO (eq. 22) - Four interferers at $10 \%$ intercell power.

an outage of almost $2 \%$. In the sub-cooperative conditions the AV-MIMO-SCO system achieves a channel capacity of 25 bits/s/Hz outperforming the saturated cooperative by almost $10 \mathrm{bits} / \mathrm{s} / \mathrm{Hz}$ at $30 \mathrm{~dB}$ of SNR.

\section{CONCLUSION}

The AV-MIMO-SCO system operating in a single cluster small cell enables interference to be controlled and allows results comparable to the theoretical upper bound for transmission of bid data. This is important factor for high spectral efficient cooperative systems, particularly when interference is unknown where traditional cooperative systems will fail. This is expatiated in the results where in sub-cooperative environments the cooperative MIMO system saturates in fairly high BER while spectral efficiency is approximately $10 \mathrm{bit} / \mathrm{s} / \mathrm{Hz}$ (at $\mathrm{SNR}=30 \mathrm{~dB}$ ) less than the sub-cooperative AV-MIMOSCO approach. It can be further noted that in a cooperative environment the two systems perform similarly, within $1 \mathrm{~dB}$ of SNR in BER and $0.5 \mathrm{bits} / \mathrm{s} / \mathrm{Hz}$ in spectral efficiency.

Furthermore, the AV-MIMO-SCO system is a completely decentralized approach needless of a coordinating node to either cooperative and sub-cooperative mobile environments while it performs equally well under partial or no channel state information. Finally, due to it dependency on AMUD, AV-MIMO-SCO enable linear computational complexity when increasing the access nodes and unknown interferers of the overall system.

\section{REFERENCES}

[1] E. Telatar, "Capacity of Multi-antenna Gaussian Channels", European Trans. Telecomm. ETT, vol. 10, pp. 585-596, Nov/Dec 1999.

[2] G. J. Foschini, M. J. Gans, "On limits of wireless communications in a fading environment when using multiple antennas", Wireless Personal Communications, vol. 6, pp. 311-335, 1998.

[3] A. J. Paulraj, C. B. Papadias, "Space-time processing for wireless communications", IEEE Signal Processing Magazine, vol. 14, Iss. 6, pp. 49-83, Nov 1997.

[4] A. Goldsmith, S. A. Jafar, N. Jindal, S. Vishwanath, "Capacity limits of MIMO channels", IEEE Journal on Selected Areas in Communications, vol. 21, Iss. 5, pp. 684-702, June 2003.

[5] D. S. Shiu, G. J. Foschini, M. J. Gans \& J. M. Kahn, "Fading Correlation and Its Effect on the Capacity of Multielement Antenna Systems", IEEE Trans. on Communications, vol. 48, No. 3, pp. 502-513, Mar 2000.

[6] T. Kanakis, C. Litchfield, P. Rapajic, "Adaptive Virtual Relaying MIMO; Decode and Forward", WCSP 2009, Nanjing, China, Nov 2009.

[7] S. Verdu, "Multiuser Detection", Cambridge University Press, 2003.

[8] P. B. Rapajic and D. K. Borah, "Adaptive MMSE maximum likelihood CDMA multiuser detection", IEEE Journal on Selected Areas in Communications, vol. 17, Iss. 12, pp. 2110-2122, Dec 1999.

[9] P. B. Rapajic and B. S. Vucetic, "Adaptive Receiver Structures for Asynchronous CDMA Systems", IEEE Journal on Selected Areas in Communications, vol. 12, No. 4, pp. 685-697, May 1994.

[10] M. Dohler, E. Lefranc \& H. Aghvami, "Virtual Antenna Arrays for Future Wireless Mobile Communication Systems", ICT, China, Jun 2002.

[11] M. Dohler, E. Lefranc \& H. Aghvami, "Link Capacity Analysis of Virtual Antenna Arrays", VTC Fall, Vancouver, Canada, Sept 2002.

[12] V. D. Trajkovic, P. B. Rapajic, R. A. Kennedy, "Adaptive ordering for imperfect successive decision feedback multiuser detection", IEEE Transactions on Communications, vol. 56, Iss. 2, pp. 173-176, Feb 2008.

[13] C. Shannon, "A mathematical theory of communication", Bell Syst. Tech. J., vol.27, pp. 379-423, 623-656, July-Oct. 1948.

[14] T. Yoo, E. Yoon, A. Goldsmith, "MIMO Capacity with Channel Uncertainty: Does Feedback Help?", IEEE Globecom 2004, Dalas, Dec 2004.

[15] A. Papoulis, S. U. Pillai, "Probaility, Random Variables and Stochastic Processes", Fourth Edition, McGraw Hill, 2002.

[16] S. Verdu, "Minimum Probability of Error for Synchronous Gaussian Multiple-Access Channels", IEEE Trans. on Information Theory, vol. 32, Iss. 1, pp. 85-96, Jan 1986.

[17] U. Madhow, M. L. Honig, "MMSE interference suppression for directsequence spread-spectrumCDMA", IEEE Trans. on Communications, vol. 42, Iss. 12, pp. 3178-3188, Dec 1994.

[18] S. Verdu, S. Shamai, "Spectral efficiency of CDMA with random spreading", IEEE Trans. on Information Theory, vol. 45, Iss. 2, pp. 622 640, Mar 1999

[19] H. V. Poor, S. Verdu, "Single-user Detectors for Multiuser Channels", IEEE Trans. on Communications, vol. 36, No. 1, pp. 50-60, Jan 1988.

[20] T. M. Cover, A. A. Elgamal, "Capacity Theorems for the Relay Channel", IEEE Trans. on Information Theory, vol. 25, Iss. 5, pp. 572-584, Sept 1979.

[21] P. Gupta, P. R. Kumar, "The capacity of wireless networks", IEEE Trans. on Information Theory, vol. 46, Iss. 2, pp. 388-404, Mar 2000.

[22] G. Kramer, M. Gastpar, P. Gupta, "Cooperative Strategies and Capacity Theorems for Relay Networks", IEEE Trans. on Information Theory, vol. 51, Iss. 9, pp. 3037-3063, Sept 2005.

[23] A. Sendonaris, E. Erkip, B. Aazhang "User cooperation diversity, Part I, II', IEEE Trans. on Communications, vol. 51, Iss. 11, pp. 1927-1948, Nov 2003.

[24] J. N. Laneman, D. N. C. Tse, G. W. Wornell, "Cooperative diversity in wireless networks: Efficient protocols and outage behavior", IEEE GLOBECOM '02, pp. 17-21, Nov 2002.

[25] J. N. Laneman, G. W. Wornell, "Distributed space-time coded protocols for exploiting cooperative diversity in wireless networks", IEEE Trans. on Information Theory, vol. 50, Iss. 12, pp. 3062-3080, Dec 2004.

[26] A. Nosratinia, T. E. Hunter, A. Hedayat "Cooperative communication in wireless networks", IEEE Communications Magazine, vol. 42, Iss. 10, pp. $74-80$, Oct 2004 